\title{
A Better EFGM-FEM Direct Coupling Method
}

\author{
Yujing Gao \& Dehua Wang \\ Shandong Vocational College of science \& Technology \\ Weifang 261100, Shandong, China \\ E-mail: wangdehua2000@163.com
}

\begin{abstract}
Coupling method is developed in recent years to solve numerical problems a new method, meshless - the finite element of a direct coupling method is based on the definition of the generalized unit of coupling of the new method. The core of this method is the use of each unit in the shape function to the assumption that the brain that the whole sub-domain to be seeking to solve the unknown field function. Coupling with other compared with the method is simple to calculate the advantages of a short time.
\end{abstract}

Keywords: EFGM-FEM, Coupling method

\section{Introduction}

The basic idea of meshless and finite element is to divide successive solve domain into limited conbanation unit that was connected according to a certain way. Units in meshless method only have meanings in number. It is different from the unit in limited element. When forming cell shape function, finite element method is obtained through the nodes of the interpolation unit. Meshless method is obtained through using mobile least square method to the node of the unit or using core function and other methods. We unify the meshless method and a direct coupling method through defining a kind of the coupling unit. To define the unit that has numerical meaning of the finite element method and meshless method. Namely, to use the assumption that each unit of the shape function to the brain that the whole sub-domain to be seeking to solve the unknown field function. Meshless method and finite element method only have difference when solving the shape function in unit. Both of them can be seen as different kinds of broad definition of the coupling unit.

\section{Based on a broad definition of the coupling unit - meshless and finite element direct coupling method}

To divide the solve domain using finite element unit and non grid unit, the obtained discrete model is the system potential energy of the various units and the potential energy, that is

$$
\prod_{\rho}=\sum_{e}\left(a^{e T} \int_{\Omega_{e}} \frac{1}{2} B^{T} D B t d x d y a^{e}-\sum_{e}\left(a^{e T} \int_{\Omega_{e}} N^{T} f t d x d y\right)-\sum_{e}\left(a^{e T} \int_{\Omega_{e}} N^{T} T t d x d y\right)\right)
$$

The structure node displacement of $a$ replace the displacement unit node of $a^{e}$

$a=C a$, at the same time $\int_{\Omega_{e}} \frac{1}{2} B^{T} D B t d x d y=K^{e}$,

$\left.\left.\int_{\Omega_{e}} N^{T} f t d x d y\right)=M^{e}, \int_{\Omega_{e}} N^{T} T t d x d y\right)=Q^{e} .(1)$ fumular can be changed

$$
\begin{gathered}
\prod_{\rho}=\sum_{e}\left((C a)^{T} K^{e}(C a)\right)-\sum_{e}\left((C a)^{T} M^{e}\right)-\sum_{e}\left((C a)^{T} Q^{e}\right) \\
=a^{T} \sum_{e}\left(C^{T} K^{e} C\right) a-a^{T} \sum\left(C^{T} M^{e}\right)-a^{T} \sum\left(C^{T} Q^{e}\right) \\
=a^{T} \sum_{e}\left(C^{T} K^{e} C\right) a-a^{T} \sum\left(C^{T}\left(M^{e}+Q^{e}\right)\right)
\end{gathered}
$$

Because of $\delta \prod_{\rho}=0$,so we can get hold of an equation

$$
K a=P
$$

In this fomular, $K=\sum_{e}\left(C^{T} K^{e} C\right), P=\sum_{e}\left(C^{T} P^{e}\right)$.

$\mathrm{C}$ is the transition matrix element node it decide the position element stiffness matrix in stiffness matrices.

Attention: in the specific calculation, we should calculated, respectively, the finite element stiffness matrix region. $K_{1}$ and $K_{2}$ stiffness matrix in non grid region. Then according to the overall number of nodes, put $K_{1}$ and $K_{2}$ in the stiffness matrices, and solve the stiffness matrices£and slove global offset.

This passage take the finite element as an example to explain the entire process of putting element stiffness matrix in the stiffness matrices. For example 1-1, assuming there is a simple planar structure. To chose six nodes, divided into four 
modules. The number of the unit and nodes are pictures 1-1,Each node has two degrees of freedom, the process of install stiffness matrices:

$$
\prod_{\rho}=a^{T} \sum_{e}\left(C^{T} K^{e} C\right) a-a^{T} \sum_{e}\left(C^{T} P^{e}\right)
$$

Because of $\delta \prod_{\rho}=0$,so we can get hold of an equation

$$
K a=P
$$

In this fomular, $K=\sum_{e}\left(C^{T} K^{e} C\right), P=\sum_{e}\left(C^{T} P^{e}\right)$.

$\mathrm{C}$ is the transition matrix element node it decide the position element stiffness matrix in stiffness matrices.

Attention: in the specific calculation, we should calculated, respectively, the finite element stiffness matrix region. $K_{1}$ and $K_{2}$ stiffness matrix in non grid region. Then according to the overall number of nodes, put $K_{1}$ and $K_{2}$ in the stiffness matrices, and solve the stiffness matrices£and slove global offset.

This passage take the finite element as an example to explain the entire process of putting element stiffness matrix in the stiffness matrices. For example 1-1, assuming there is a simple planar structure. To chose six nodes, divided into four modules. The number of the unit and nodes are pictures 1-1, Each node has two degrees of freedom, the process of install stiffness matrices:

(1) According to the order of units of local number just matrix-forming units. Picture 1-1 unit(3), Nodes in order of local number $1, \mathrm{~m}, \mathrm{n}$.

Newly formed units to sub-matrix matrix form is given for the

$$
\left.[K]^{3}=\underset{n}{l}=\begin{array}{ccc}
l & m & n \\
K_{l l} & K_{l m} & K_{l n} \\
K_{m l} & K_{m m} & K_{m n} \\
K_{n l} & K_{n m} & K_{n n}
\end{array}\right)
$$

(2) To change the unit of the local node number $(l, m, n)$ into total numberfjust of the corresponding matrix element of the subscript of the matrix is also replaced by the overall number. To convert the stiffness matrix of Picture 1-1 unit (3) after the overall number is

$$
\begin{gathered}
l(5) \\
{[K]^{3}=\underset{m(3)}{m(2)}}
\end{gathered}\left(\begin{array}{lll}
K_{55} & K_{53} & K_{52} \\
K_{35} & K_{33} & K_{32} \\
K_{25} & K_{23} & K_{22}
\end{array}\right)
$$

(3) Put each sub-matrix of element stiffness matrix that was converted into the corresponding position of the the overall stiffness matrix. Put each sub-matrix of unit (3) into

$$
\begin{aligned}
& \begin{array}{llllll}
1 & 2 & 3 & 4 & 5 & 6
\end{array}
\end{aligned}
$$

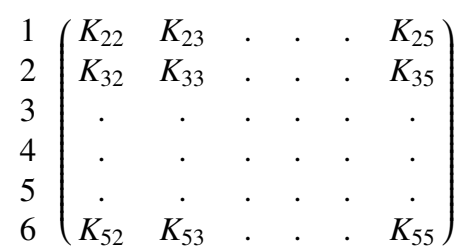

(4) After putting all the unit into carry out(1), (2), (3)step, we can obtain the stiffness matrices, as it mentioned above

$$
\left[K_{1}\right]=\left(\begin{array}{cccccc}
K_{11}^{1} & K_{12}^{1} & K_{13}^{1} & & & \\
K_{21}^{1} & K_{22}^{1+2+3} & K_{23}^{1+3} & K_{24}^{2} & K_{25}^{2+3} & \\
K_{31}^{1} & K_{32}^{1+3} & K_{33}^{1+3+4} & & K_{35}^{3+4} & K_{36}^{4} \\
& K_{42}^{2} & & K_{44}^{2} & K_{45}^{2} & \\
& K_{52}^{2+3} & K_{53}^{3+4} & K_{54}^{2} & K_{55}^{2+3+4} & K_{56}^{4} \\
& & K_{63}^{4} & & K_{65}^{4} & K_{66}^{4}
\end{array}\right)
$$

The upper right corner of $K_{l m} \mathrm{~S}$ is the sub-matrix that was cumulated on the units. we can see that the sub matrix $\mathrm{AB}$ in the matrix of element stiffness matrix is the subscript $1, \mathrm{~m}$ those of the cumulative matrix after converting matrix into the overall matrix. 
We can got the element stiffness matrix $K_{1}$, and we can got $K_{2}$ using the same method, We use local number when we solve the $K_{1}$ and $K_{2}$. We use overall number when we solve $K$. The shared nodes of finite element and non grid has two local numbers and only has one overall number. There appears Superposition when assemble the total.

\section{The weight function}

According to the general principle of choosing the weight function, there are many weight functions that can meet the conditions. Power weight function has the higher accuracy and was commonly used.

$$
w\left(d_{I}\right)=\left\{\begin{array}{cc}
\frac{e^{-\left(d_{I} / c\right)^{2}}-e^{-\left(d_{m} / c\right)^{2}}}{1--e^{-\left(d_{m} / c\right)^{2}}} & d_{I} \leq d_{m} \\
0 & d_{I}>d_{m}
\end{array}\right.
$$

In this fomular, $c$ is a constant control,it decided the shape of weight function, define the $c$ as

$$
c=\alpha c_{I}
$$

In this fomular, $1 \leq \alpha \leq 2$, and

$$
c_{I}=\max \left\|x_{J}-x_{I}\right\|
$$

In this fomular, $S_{J}$ is the minium arrange of polygon forming by $x_{I}$ and neighboring points. When nodes in the area are formly distribued, $c_{J}$ check the maximum distance between nodes, when nodes in the area are not formly distributed, $c_{I}$ points included $x_{I}$ in the regional node of the characteristic length.

$d_{m}$ is the greatest radius of the domain supported by Weight function, at the same time

$$
d_{m}=\beta c
$$

In this fomular, $\beta$ is the constant. In different problem, the value of the $\beta$ can be different.

\section{The example}

Example 1 A 100mm300mm rectangular plate is illus-trated in Fig.1-2(a), and Fig. 1-2(b) is the figure of the plate which discreted by EFGM-FE. The bottom of the rectangular plate is fixed along direction, the top of the rectangular plate is subjected to a regular tension force of $100 \mathrm{MPa}$. Discrete form as follows: upper and lower ends is the eight-node is oparametric element,respectively, the middle of the domain is represented by a set of equalized distributed meshless nodes. The material properties as follows: Young's modulus: $E=202000 M P a$,Poisson's ratio: $\mu=0.3$.

Based on the Integral Program introduced by literature (Krongauz Y, 1996), we use $2 \times 2$ the background cells in meshless domain and the fourth-order Gaussian integration, Shape function using quadratic basis functions obtained radius of influence domain by self-adaptive method, the Compactly supported control factor of weight function is $\beta=5.0$. The following table 1-1 shows the comparison between the calculated result with the theoretical solution for the displacement of the nodes.

From the above table, we can see that the EFGM-FEM Direct Coupling Method solution can be very good only when where are 9 nodes in meshless domain.

Example 2 Geometric structure, Constraints and load type are exactly the same with the example 1,and there is edge cracks in. $y=150$ only. We use $4 \times 4$ isoparametric element in the finite element domain and distribute 88 nodes in meshless domain,intensify the cracks. The distribution of Meshless nodes and integration mesh are shown as Figure 1-3 (a) and Figure 1-3 (b).We use quadratic basis functions, the number of nodes in influence domain is 40,the Compactly supported control factor is 5.0,Diffraction theorem is used to deal with crack discontinuities.

Fig. 1-3 The distribution of Meshless nodes and integration mesh when the length of the crack is $50 \mathrm{~mm}$

By direct displacement method,we can calculated the non-dimensional stress intensity factor $F=K_{1} / \sigma \sqrt{\pi a}$ when crack length is $0 \mathrm{~mm}, 10 \mathrm{~mm}, 20 \mathrm{~mm}, 30 \mathrm{~mm}, 40 \mathrm{~mm}, 50 \mathrm{~mm}, 60 \mathrm{~mm}$.According to the literature (Chinese Aeronautical Establishment, 2007), the expression of given by

$$
F=1.12-0.23 \frac{a}{b}+10.6\left(\frac{a}{b}\right)^{2}-21.7\left(\frac{a}{b}\right)^{3}+30.4\left(\frac{a}{b}\right)^{4}
$$

Where $a$ is the length of the crack, $b$ is the width of the ban. The Fig.1-4 shows a comparison between calculated solution and the theoretical solution.

\section{Conclusion}

In this paper,A brief description of the basic theory of EFGM-FEM Direct Coupling Method is pro-posed. Meshless-finite element numerical of the direct coupling method does not require structural shape function for transition region neither 
require the Lagrange multipliers, therefore, this method has the advantages of flexible operations and small calculated amount. Numerical examples show high accuracy and have bright application prospects.

\section{References}

Belytschko T, Lu Y Y. and Gu L. (1994). Element-Free Galerkin Methods. Int J Numer Methods Engrg, 37, $229-256$.

Belytschko T, Organ D, and Krongauz Y. (1995). A Coupled Finite Element-Element-Free-Galerkin Method. Computer Mechanics, 17, 186-195.

Chinese Aeronautical Establishment. (2007). The hand-book about stress intensity factor. BeJing: Science Press, (Chapter 3).

Hegen D. (1996). Element-Free Galerkin Method in Combination with Finite Element Approaches. Compu Methods Appl Mech Engrg, 135, 143-146.

JIA-liang, HUANG-qiqing, and YIN-zhiping. (2007). EFGM-FEM Direct Coupling Method. Journal of Northwestern Polytechnical University. 337-341.

Krongauz Y, Belytschko T. (1996). Enforcement of Essential Boundary Conditions in Meshless Approximations Using Finite Elements. Compu Methods Appl Mech Engrg, 131, 133-145.

Lancaster P, Salkauskas K. (1981). Surfaces Generated by Moving Least-Squares Methods. Math Computer, 37, $141-158$.

Table 1. The comparison of the EFGM-FEM Direct Coupling Method solution and the theoretical solution

\begin{tabular}{cccc}
\hline $\mathrm{y}$ & $\mathrm{u}$ _y Numerical sol & Theoretical Sol & Relative error/\% \\
\hline 50 & 0.0250555 & 0.0247525 & 1.22 \\
100 & 0.0501352 & 0.0495050 & 1.27 \\
150 & 0.0742761 & 0.0742574 & 0.03 \\
200 & 0.0983749 & 0.0990099 & -0.64 \\
250 & 0.123630 & 0.1237624 & -0.11 \\
300 & 01488980 & 0.1485149 & 0.26 \\
\hline
\end{tabular}

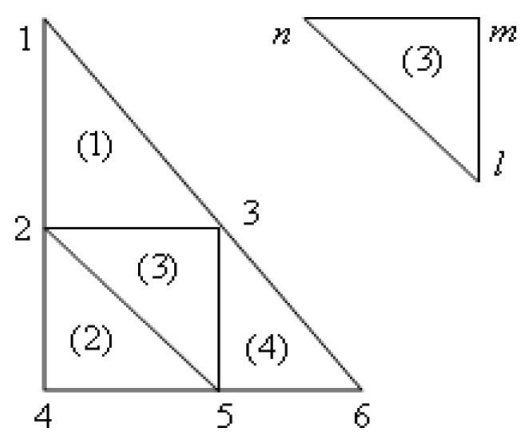

Figure 1. 

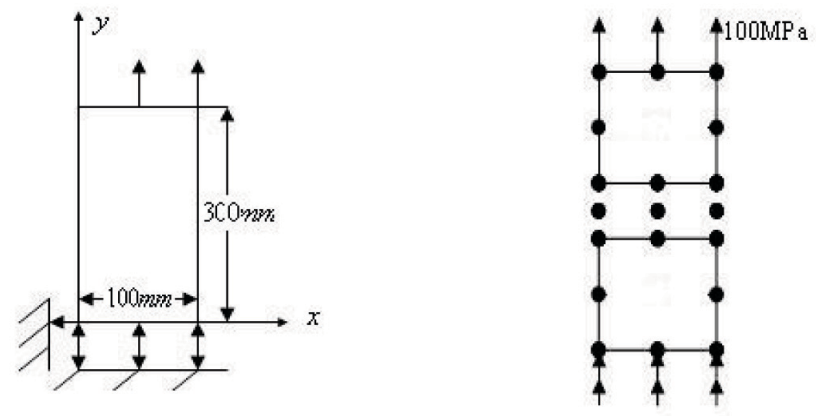

(a)Simply supported beam figure (b)Discrete figure

Figure 2. Simply supported beam figure and discrete figure of rectangular plate

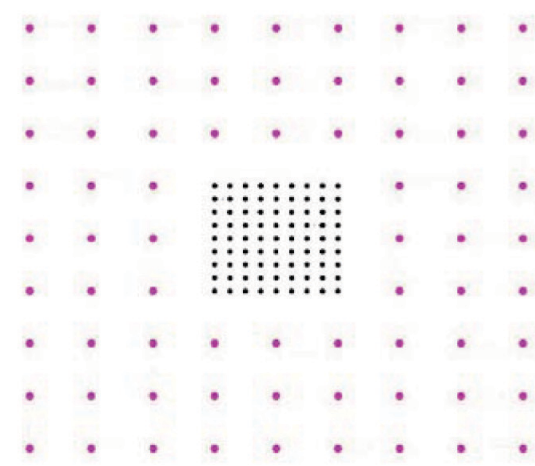

(a)The distribution of Meshless nodes

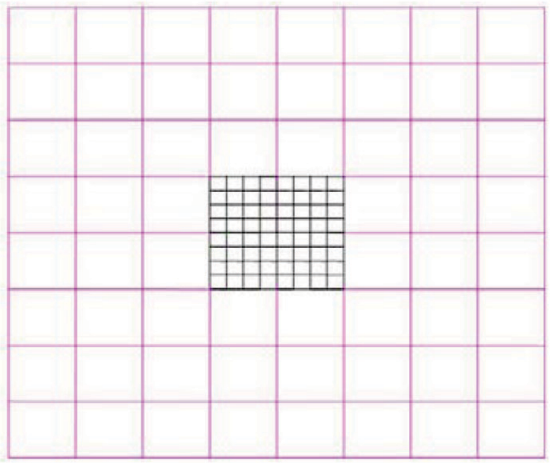

(b) Integration mesh

Figure 3. The distribution of Meshless nodes and integration mesh when the length of the crack is $50 \mathrm{~mm}$

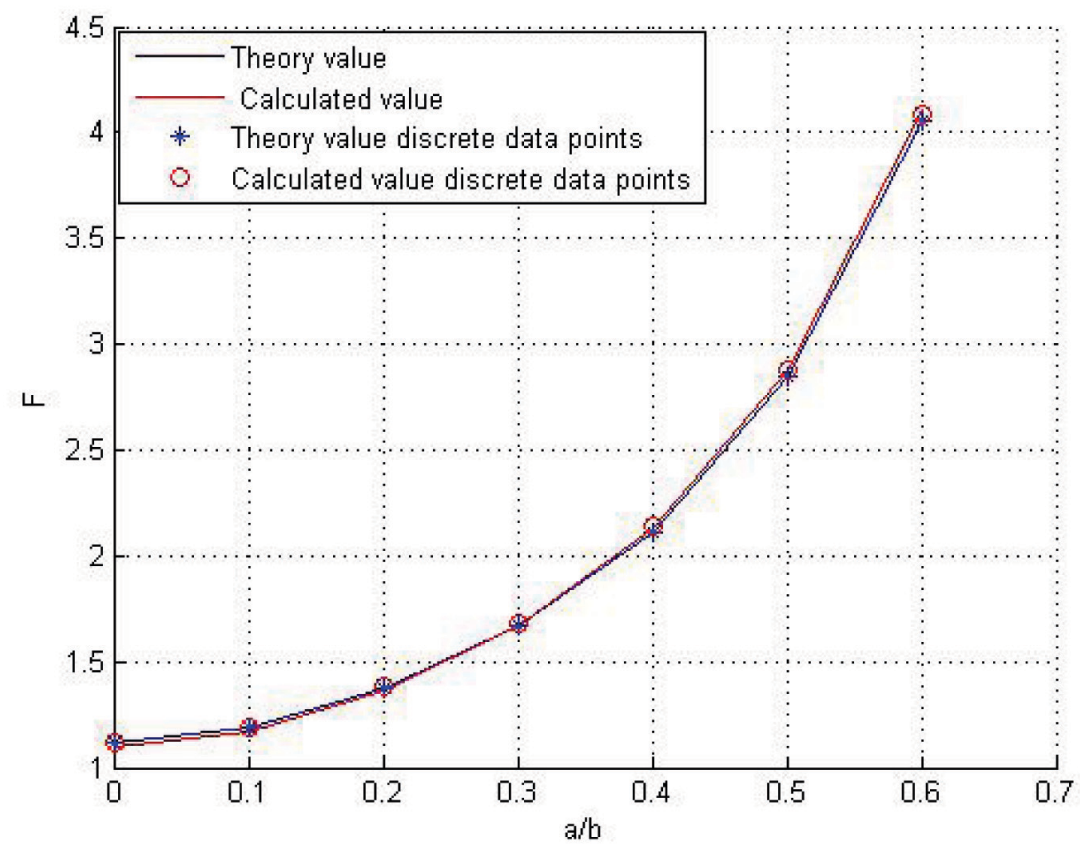

Figure 4. Comparison of the calculated solution and the theoretical solution 